\title{
El valor de la comunicación organizacional: una visión desde los profesionales, académicos y consultores
}

\section{The value of organizational communication: a vision from professionals, academics and consultants}

Rebeca Illiana Arévalo Martínez*

Universidad Anáhuac México

Av. Universidad Anáhuac núm. 46, Col. Lomas Anáhuac, Huixquilucan, Estado de México, C.P. 052786, México

Editor: Rogelio del Prado Flores

Fecha de recepción: 9 de octubre de 2021

Fecha de aceptación: 10 de diciembre de 2021 rebeca.arevalo@anahuac.mx https://orcid.org/0000-0003-1163-6752

\section{RESUMEN}

El presente artículo muestra los resultados de una investigación cualitativa que tuvo por objetivo analizar la conceptualización que se tiene respecto al capital económico, cultural y social de las organizaciones de voz de los profesionales, consultores y académicos en comunicación organizacional con el fin de vislumbrar la contribución que pueden tener desde su función a la formación de dichos capitales. La muestra estuvo constituida por 30 personas en México, quienes ocupan una posición en las organizaciones en áreas de comunicación o bien son académicos o consultores en comunicación organizacional. Entre los principales resultados se encontró la necesidad de homologar el concepto de capital social, la ausencia de referencia al capital cultural entre los profesionales que trabajan en las organizaciones y el énfasis en el capital económico entre los académicos.

Palabras clave: comunicación organizacional, capital, social, cultural, económico.

\footnotetext{
* Directora del Centro de Investigación para la Comunicación Aplicada (CICA) y Coordinadora Académica del Doctorado en Investigación de la Comunicación (PNPC-Conacyt) de la Facultad de Comunicación. Miembro del Sistema Nacional de Investigadores (SNI), Nivel I Conacyt. Coordinadora GT2 Comunicación Organizacional y Relaciones Públicas en Asociación Latinoamericana de Investigadores de la Comunicación (ALAIC). Presidenta de Asociación Mexicana de Investigadores y Profesionales de la Comunicación Organizacional (AMIPCO).
} 


\section{ABSTRACT}

This article shows the results of a qualitative research that aimed to analyze the conceptualization of the economic, cultural and social capital of the voice organizations of professionals, consultants and academics in organizational communication in order to glimpse the contribution they can have from their function to the formation of these capitals. The sample consisted of 30 people in Mexico, who occupy a position in organizations in areas of communication or are academics or consultants in organizational communication. Among the main results was the need to standardize the concept of social capital, the absence of reference to cultural capital among professionals working in organizations and the emphasis on economic capital among academics.

Keywords: organizational communication, capital, cultural, social, economic.

\section{INTRODUCCIÓN}

En el ámbito de la comunicación organizacional se reconoce que uno de sus principales propósitos es contribuir al logro de las metas de la organización; sin embargo, en la mente de quienes ejercen esta función, tanto en el ámbito profesional, de consultoría y en el académico, la conceptualización de cómo es dicha contribución no se ha homologado. En este artículo se presenta una investigación cualitativa que tuvo por objetivo analizar la conceptualización que se tiene respecto al capital económico, cultural y social de las organizaciones de manera que pueda entrelazarse con la mirada que tienen de cómo es que la comunicación puede aportar algo en su formación. La muestra estuvo constituida por 30 profesionales de comunicación en México, quienes ocupan una posición en las organizaciones en áreas de comunicación o bien son académicos o consultores en comunicación organizacional. Entre los principales resultados destaca la diferenciación en el concepto de capital social, el énfasis en las relaciones por parte de los académicos y de la ausencia del capital cultural entre los profesionales que laboran en comunicación organizacional en el interior de instituciones u organizaciones. La necesidad de homologar las visiones de estos capitales podría contribuir a afrontar los retos actuales desde la comunicación con una mayor certeza y capacidad de involucramiento con base en su conocimiento y experiencia. 


\section{MARCO TEÓRICO}

A lo largo de la evolución de la comunicación organizacional, una de las principales preocupaciones de quienes la ejercen ha sido demostrar su contribución a los resultados de la organización. En este empeño se encuentran investigaciones como la de Grunig, Grunigy Dozier (2002), quienes concluyeron que no existían indicadores cuantificables suficientes para ponderar la calidad de la comunicación en las organizaciones a partir de las relaciones que establecen en el largo plazo, y es por ello por lo que más adelante propusieron cuantificar los ahorros producto de la comunicación efectiva y su impacto en la reducción de riesgos para la organización (Grunig, J. y Grunig, L., 20II).

Derivado de lo anterior se retoma el concepto de campo de Pierre Bourdieu (2002), entendido como el lugar donde se construyen los significados, donde se produce el sentido común a partir de lo que se considera adecuado o esperado y lo que no es adecuado, y con ello una lucha de fuerzas por el control del capital que es producto de dicho campo (Bourdieu, 2003a). Es así como el capital se define como este valor que se produce en determinado campo y por el cual luchan los agentes que están dentro de él. El capital puede ser económico cuando se trata de recursos financieros o monetarios (Bourdieu, 2003), social cuando emana de las relaciones o redes que se conforman en el campo (Bourdieu, 2007), o cultural cuando es el resultado de disposiciones o hábitos adquiridos por la relación en una determinada clase social (Bourdieu, I996).

Cabe señalar que la organización se concibe desde una perspectiva sistémica (Ackoff, 2000) en la cual se inserta justamente la comunicación de manera intrínseca en el proceso de síntesis a nivel interno en la relación e interacción entre las partes que conforman al sistema (subsistema), y en el proceso de análisis al darle sentido a la participación de cada uno de estos subsistemas y crear un contacto continuo entre ellos (Barquero y Castillo-Esparcia, 20II). Asimismo, la comunicación posibilita la integración de todos los subsistemas para hacer frente al exterior de manera conjunta, lo cual fortalece y da vida al papel de la organización en la comunidad, dentro de la cual busca sinergias (De Salas y Pérez, 20Io) a fin de lograr el acuerdo en el desacuerdo y la armonía entre la disonancia que implica la complejidad (Etkin, 2006) en la que se desenvuelve y de la cual forma parte.

La teoría sistémica que brindan Daniel Katz y Robert Kahn (1966) sirve de marco para la definición de organización como un sistema abierto dentro del cual la comunicación es descentralizada y la retroalimentación es muy importante porque permite el control del sistema. En este marco, la lucha por los capitales de los que habla Bourdieu (1997, 2003, 2007) dentro del campo se hace más relevante y la comunicación funge un papel primordial para posibilitar la obtención de los mismos dentro de la organización, dentro del control que 
puede ejercer en dicho sistema. Este enfoque sistémico permite explicar la forma en que se coordinan dentro y fuera del sistema los distintos elementos, ya que "los sistemas sociales operan en un medio ambiente social y físico. Las condiciones del medio ambiente tanto afectan como son afectadas por el comportamiento de los sistemas que contiene" (Ackoff, 2005, p. 87). La comunicación ayuda a que la organización cumpla con sus finalidades de servir a la comunidad, desarrollar a las personas que la integran, tener continuidad y generar un valor económico agregado (Llano, 1997), que bien podrían ser entendidos como la obtención de los capitales cultural, social y económico.

Finalmente, las acciones de comunicación que se llevan a cabo en una organización responden a la visión de quien las planea, en este sentido la comunicación está determinada por el babitus de quien la diseña, el cual se puede definir como un "principio de un conocimiento sin conciencia, de una intencionalidad sin intención y de un dominio práctico de las regularidades del mundo que permite adelantar el porvenir sin tener ni siquiera necesidad de presentarlo como tal" (Bourdieu, I996b, p. 24). Es decir, el babitus genera una forma de hacer las cosas dictadas dentro de un determinado contexto social, es una serie de reglas que influyen en la toman de decisiones dentro de la comunicación y por tanto de los capitales que se forman entre hombres y mujeres, personas con una determinada escolaridad, con estudios de comunicación de otras áreas del conocimiento, con experiencia docente o profesional o de consultoría, etc. Así, las nociones de lo que significa cada uno de los distintos tipos de capital también es diferenciada a partir del babitus de la persona en cuestión.

\section{METODOLOGÍA}

Esta investigación se realizó a partir de una metodología cualitativa a través de una entrevista estructurada que tuvo como objetivo analizar la conceptualización que se tiene respecto a la contribución que desde la comunicación se hace al capital económico, cultural y social de las organizaciones de voz de quienes lo ejercen. La muestra estuvo constituida por 30 profesionales de comunicación en México, quienes ocupan una posición en las organizaciones, son académicos o consultores en el ámbito de la comunicación organizacional (Tabla I).

La guía de entrevista estructurada con preguntas específicas en donde el entrevistador se sujeta exclusivamente a estas a fin de explorar las visiones del profesional, académico o consultor de comunicación organizacional con respecto a las categorías del concepto de capital social, cultural y económico a las organizaciones y cómo es la contribución de la comunicación a la formación de estos. La metodología que se utilizó para el análisis de las entrevistas fue en un nivel textual, contextual e interpretativo. En el nivel textual se forma- 
ron nubes de texto para identificar los principales términos o códigos utilizados en cada categoría; mientras que en el nivel contextual se hizo la búsqueda de sentido a partir de conjuntar la visión de la comunicación en la formación de cada categoría de capital (social, cultural o económico); y en el nivel interpretativo se contrastaron las respuestas con las características de cada informante en términos de las categorías de género, escolaridad y especialidad (en comunicación o en otras disciplinas).

TABLA 1. COMPOSICIÓN DE LA MUESTRA

\begin{tabular}{|c|c|c|c|c|c|c|c|c|c|c|c|c|c|c|}
\hline \multirow{2}{*}{$\begin{array}{l}\text { Organización } \\
\text { o tipo de } \\
\text { profesional } \\
\text { entrevistado } \\
\text { (cantidad) }\end{array}$} & \multicolumn{2}{|c|}{ GÉNERO } & \multicolumn{3}{|c|}{ EDAD } & \multicolumn{3}{|c|}{$\begin{array}{c}\text { ESCOLARIDAD } \\
\text { (ÚLTIMO GRADO) }\end{array}$} & \multicolumn{3}{|c|}{ ESPECIALIDAD } & \multicolumn{3}{|c|}{$\begin{array}{c}\text { AÑOS DE EXPERIENCIA } \\
\text { PROFESIONAL }\end{array}$} \\
\hline & $M$ & $\mathrm{~F}$ & $25-35$ & $36-45$ & $46-$ & LIC & MTRÍA. & $\mathrm{DR}$ & COM & $\begin{array}{l}\text { COM } \\
\text { ORG }\end{array}$ & OTRA & $1-5$ & 6-19 & 20 \\
\hline $\begin{array}{l}\text { Dentro de una } \\
\text { organización } \\
\text { (13) }\end{array}$ & 5 & 8 & 7 & 6 & & 6 & 7 & & 3 & 2 & 8 & 5 & 8 & \\
\hline Consultor(14) & 13 & 1 & 3 & 4 & 7 & 7 & 7 & & 3 & 1 & 10 & 3 & 8 & 3 \\
\hline Académico (3) & 2 & 1 & 1 & & 2 & 1 & & 2 & & 1 & 2 & & 3 & \\
\hline Totales (30) & 20 & 10 & 11 & 10 & 9 & 14 & 14 & 2 & 6 & 4 & 20 & 8 & 19 & 3 \\
\hline
\end{tabular}

FUENTE: ELABORACIÓN PROPIA.

\section{RESULTADOS}

En el análisis textual fue posible identificar la relevancia de cada uno de los capitales (cultural, simbólico y económico) en el ámbito de trabajo de los entrevistados. Cabe señalar que en los tres tipos de capital se hizo referencia a los términos organización y comunicación. En la Figura i se presenta la nube de texto que se formó con respecto al capital cultural que, como puede observarse, destaca el conocimiento, la denominación de este como un bien y la importancia del ser humano, así como la propia referencia a la palabra cultural y en menor medida la palabra cultura. Realizando un análisis interpretativo de las entrevistas es posible identificar que para los especialistas en comunicación, ya sean consultores, académicos o profesionales, el capital cultural tiene clara relación con todo aquello que constituye el co- 
nocimiento como es la información, las prácticas, mensajes, proceso, trabajo, capacidades, ideas; sin embargo, también hay grandes referencias hacia la parte colectiva, en palabras como colaboradores, contribuye, pueden, públicos, recibir, empleados, conforman, miembros, social y colectivo. Es decir, el capital cultural requiere trabajarse en comunidad, no en lo individual, su sentido se forma en su carácter colectivo y en el momento es que su desarrollo mismo forma parte de un resultado compartido. Para este fin, la comunicación tiene un papel fundamental en tejer los lazos que hagan posible esta participación conjunta que forme el capital cultural en la organización.

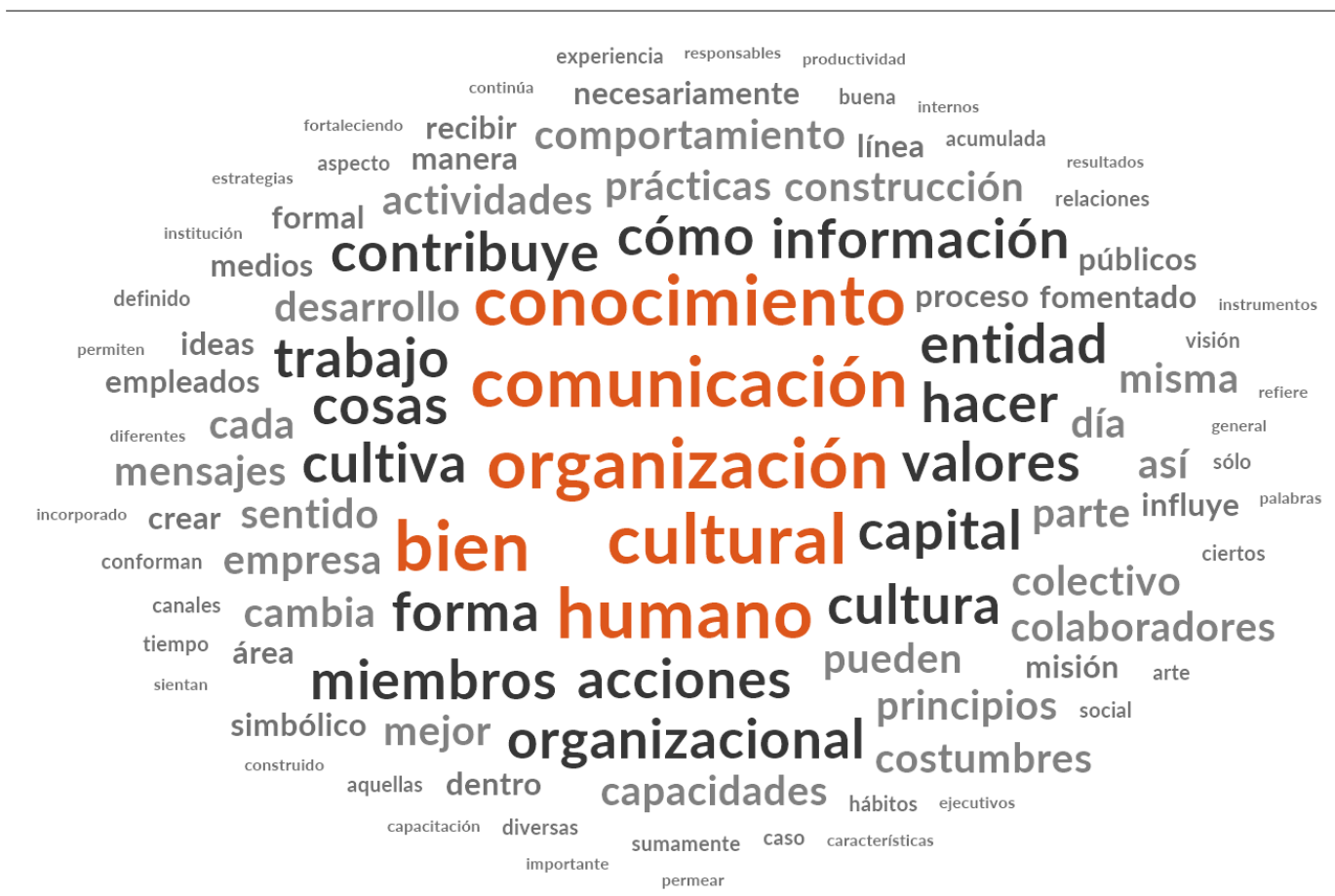

FIGURA 1. NUBE DE TEXTO SOBRE CAPITAL CULTURAL

FUENTE: ELABORACIÓN PROPIA. 
En la Figura 2 se muestran los resultados respecto al capital económico en lo que destaca la mención a los objetivos, bienes, artefactos, información, productos, resultados y la propia referencia a la palabra económico. El análisis interpretativo en cuanto al capital económico se presentan en primer lugar claramente los referentes teóricos básicos como son el capital mismo, los bienes, beneficios, productos, resultados, artefactos, materiales, recursos, ingresos, inversión, negocio e incluso la información por el valor que engloba en sí misma; sin embargo, también se encuentran referencias claras hacia los referentes que hacen posible la creación de este tipo de capital como son objetivos, acciones, e desarrollo, contribución, creación, cambio, relaciones, mecanismos, trabajo e importancia, entre otros. En este sentido, el capital económico se reviste de una serie de causas o acciones que lo posibilitan y que en general hacen referencia al esfuerzo requerido para lograrlo, dentro del cual la comunicación es una pieza clave para dar seguimiento, impulsar y coordinar acciones para el desarrollo de relaciones y el trabajo mismo requerido para cumplir con los objetivos y formar este capital.

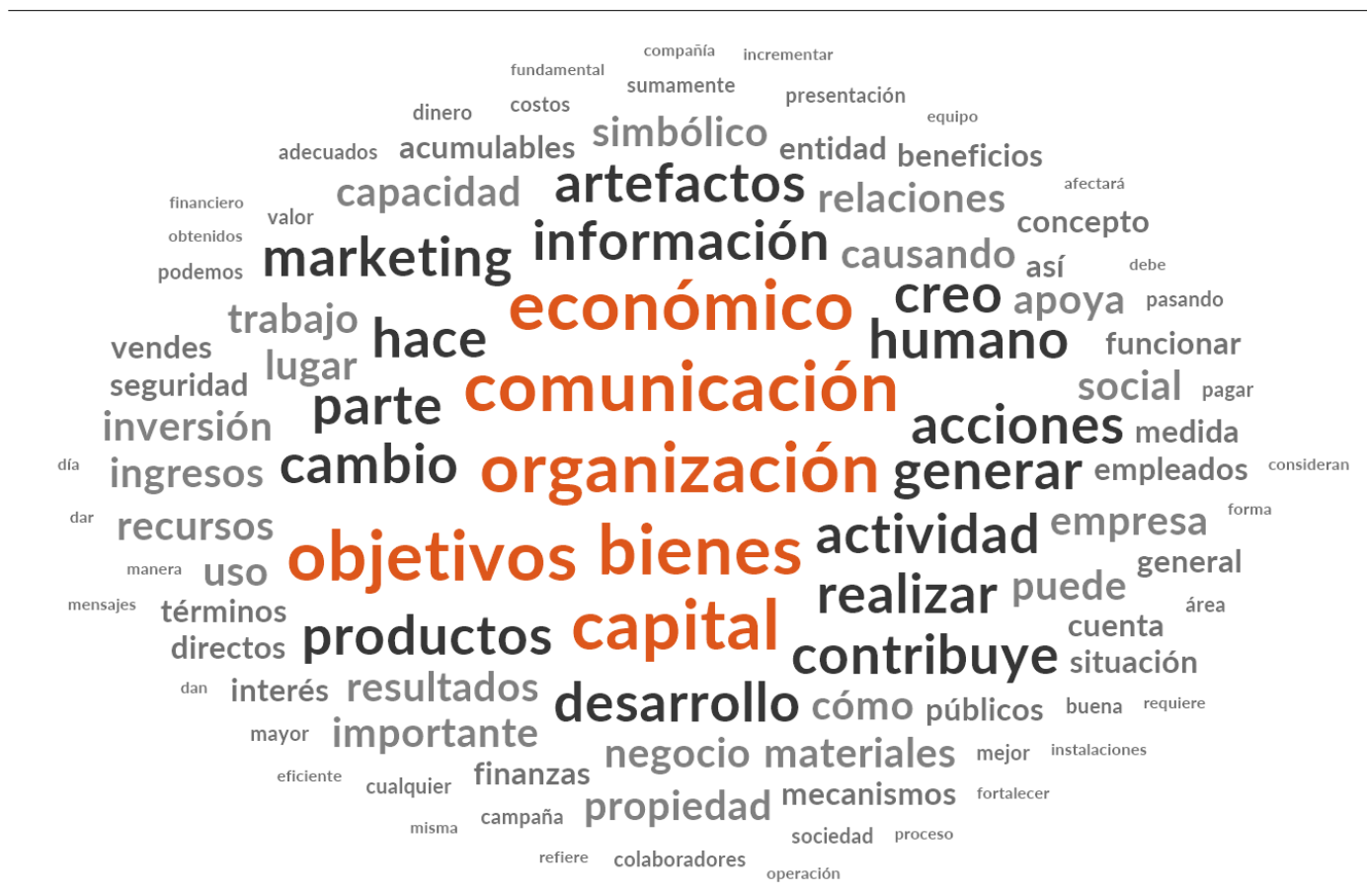

FIGURA 2. NUBE DE TEXTO SOBRE CAPITAL ECONÓMICO

FUENTE: ELABORACIÓN PROPIA. 
En el análisis textual sobre capital social que se presenta en la Figura 3 se muestra la presencia destacada de las palabras relaciones, posesión y grupos, y la propia referencia a la palabra social. En el análisis interpretativo para el caso del capital social es clara la referencia a la persona en sus distintas acepciones como miembros, colaboradores, sujetos, públicos, personas, grupos; y lo que en suma realizan las personas y que se refleja en verbos como apoyo, contribuye, causar, existen, permiten, generar, mantener, puede, cambia, buscan; o también en forma de resultados y palabras relacionadas como relaciones, logros, actividades, acciones, objetivos, finalidad, lazos, redes, afectivos, trabajo, interacción, poder, nivel, buenas. Es destacable que la palabra confianza no fuera un referente mencionado mayormente como parte del capital social, sin embargo, la comunicación se puede comprender como el eslabón que apoya, contribuye, permite, genera, mantiene los lazos, las redes y las relaciones a través de la interacción hacia los logros requeridos.

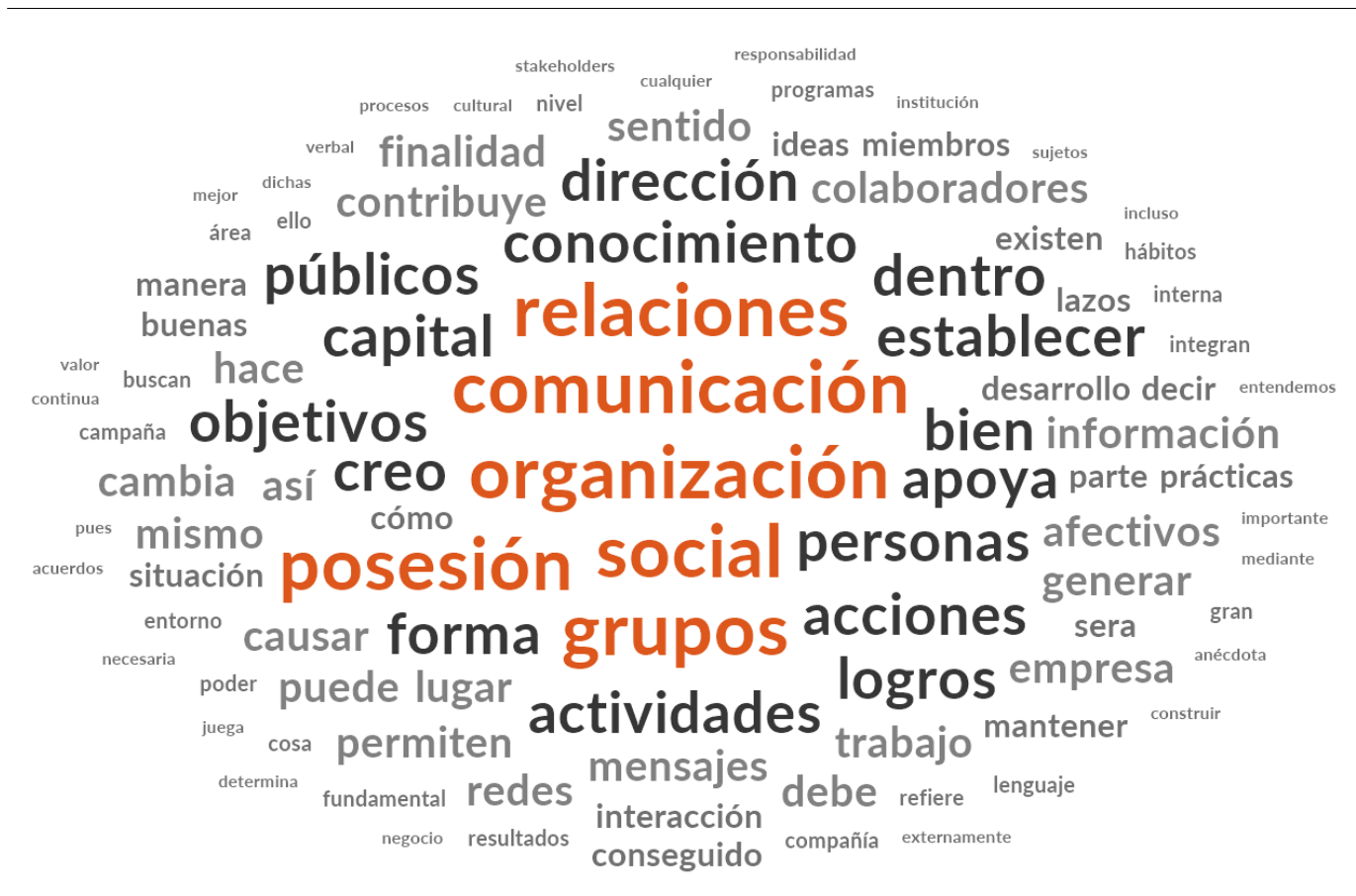

FIGURA 3. NUBE DE TEXTO SOBRE CAPITAL SOCIAL

FUENTE: ELABORACIÓN PROPIA. 
En el análisis interpretativo se contrastaron las respuestas con las características de los informantes. En primer lugar, se identificaron sus respuestas en razón de género y por ello en la Figura 4 se presentan los resultados de los entrevistados de género masculino, quienes centran sus definiciones de los distintos tipos de capital en las personas, el capital mismo, lo social y el término bien. En segundo lugar, aparecen denominadores como producción, relaciones, económico, cultura y cultural, objetivos, conocimiento, actividades, forma y acciones, entre otros. Es decir, para ellos lo más relevante de la contribución de la comunicación a los distintos tipos de capital es que las personas lo generen como un bien, destacando el capital social.

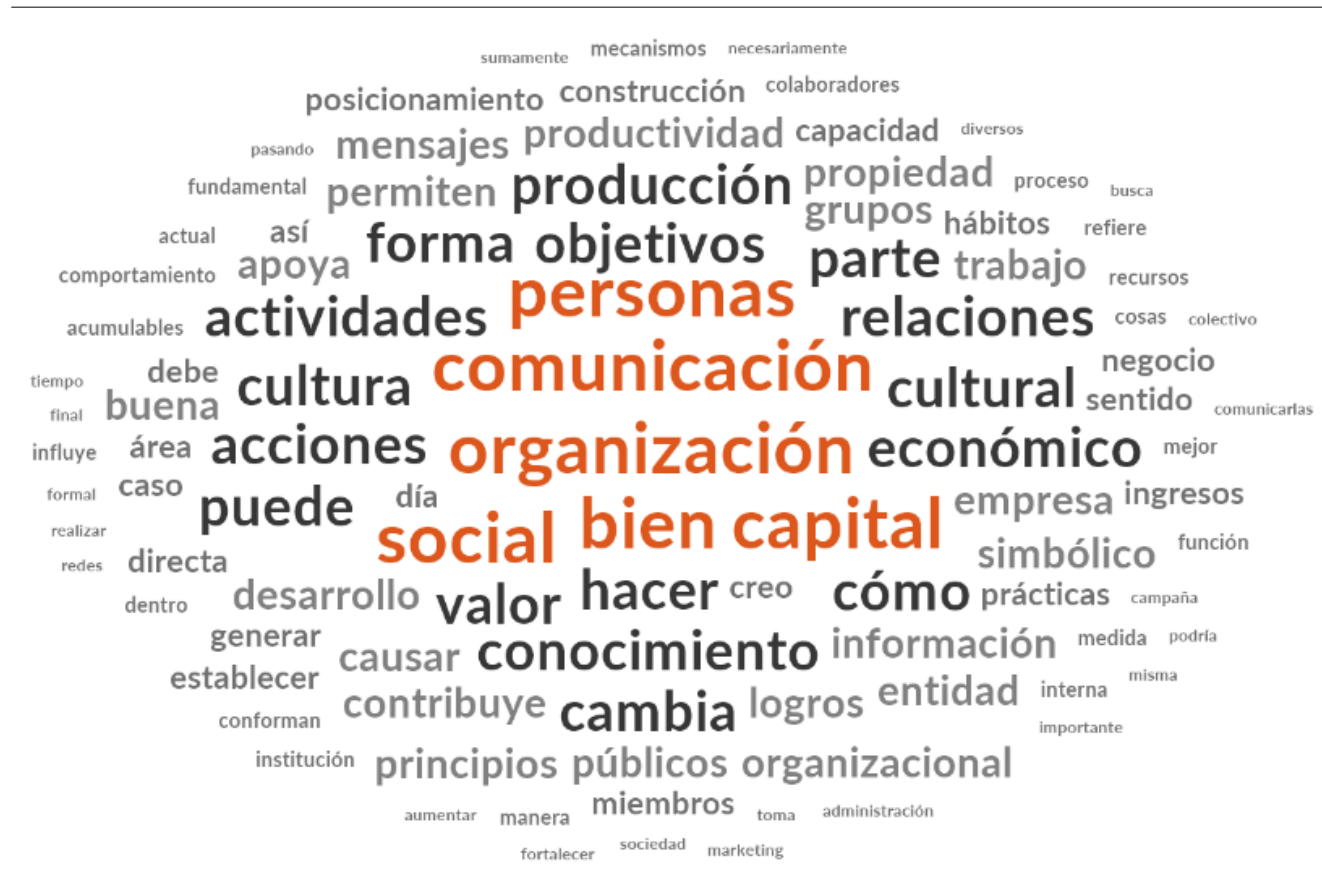

FIGURA 4. NUBE DE TEXTO SOBRE CAPITALES DE INFORMANTES DE GÉNERO MASCULINO

FUENTE: ELABORACIÓN PROPIA. 
En el caso de las entrevistadas de género femenino, como se muestra en la Figura 5, destacaron en su definición de los capitales la importancia de las relaciones, los valores, los bienes y la posesión; mencionando en segundo término aspectos como conocimiento, trabajo, objetivos, cultural, acciones, artefactos, colaboradores, empleados. Utilizaron verbos como crea, contribuye y forma, además de la referencia misma a la palabra capital. En este sentido se observa que para ellas los capitales surgen de las relaciones, pero están relacionados con el poseer bienes o valores. Es decir, a diferencia del género masculino que privilegia a las personas, el capital social y los bienes, el género femenino privilegia las relaciones, los valores y los bienes, enfatizando que se trata de una posesión, lo cual en el ámbito de la comunicación podría interpretarse como un enfoque diferenciado de su contribución a la conformación de capital social.

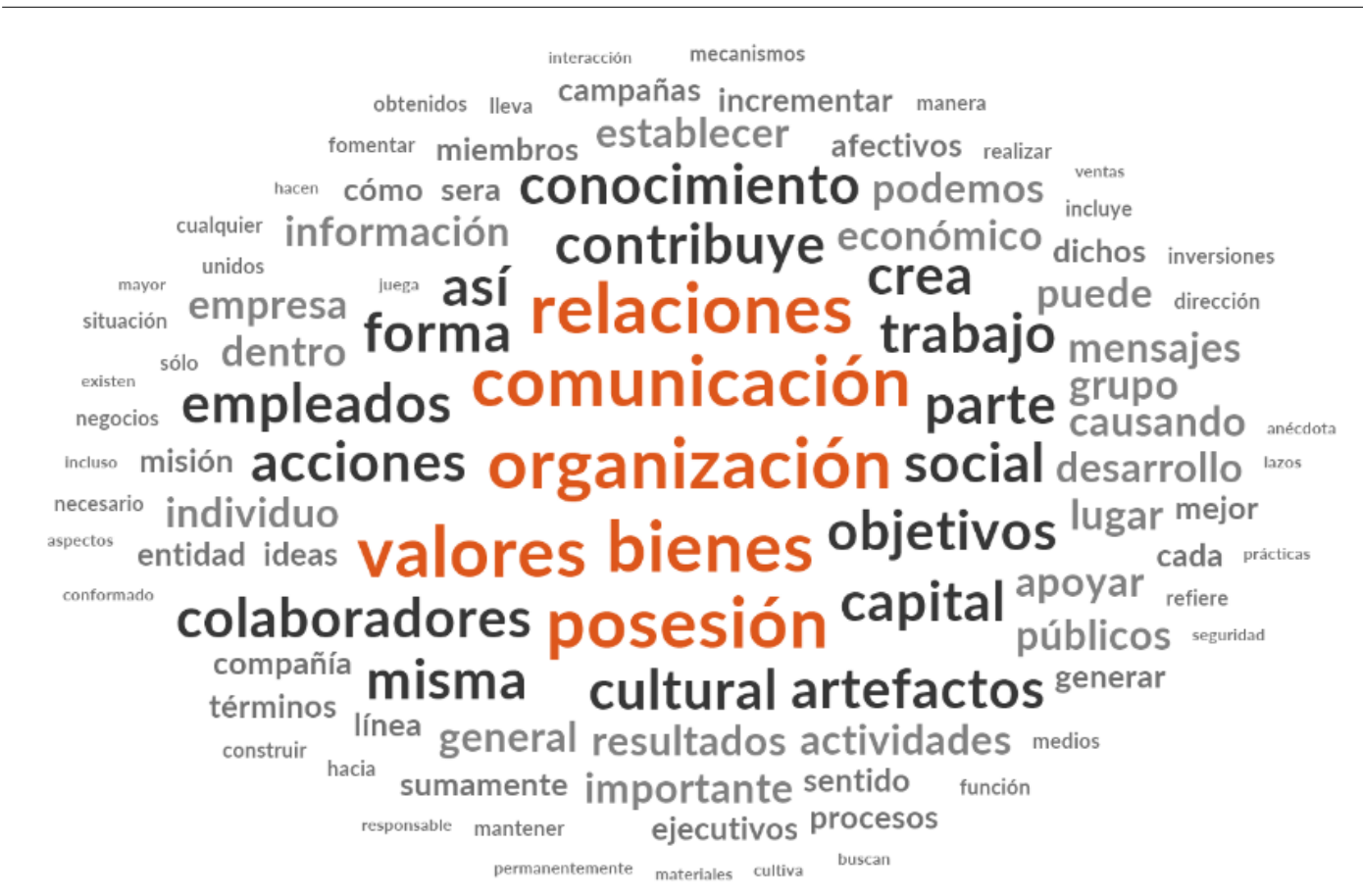

FIGURA 5. NUBE DE TEXTO SOBRE CAPITALES DE INFORMANTES DE GÉNERO FEMENINO

FUENTE: ELABORACIÓN PROPIA. 
En cuanto a la diferencia de conceptualización de los capitales en cuanto a la escolaridad, se encontró que para los entrevistados con nivel de estudios máximo de licenciatura que se muestran en la Figura 6, las definiciones están centradas en la posesión, en el bien y particularmente la referencia a la denominación de social. En segundo término, refieren lo económico y cultural, así como los objetivos, actividades, conocimiento, valores, relaciones y los verbos contribuir, hacer, formar y desarrollar. Para los entrevistados con licenciatura los capitales son concebidos como posesiones que se obtienen por acciones o actividades que realizan los individuos en la empresa a través de sus relaciones. En este sentido, la comunicación podría interpretarse que se vislumbra como el hilo conductor que permite que existan estos espacios donde las personas puedan relacionarse y por tanto contribuir al logro de objetivos o la creación de conocimiento.

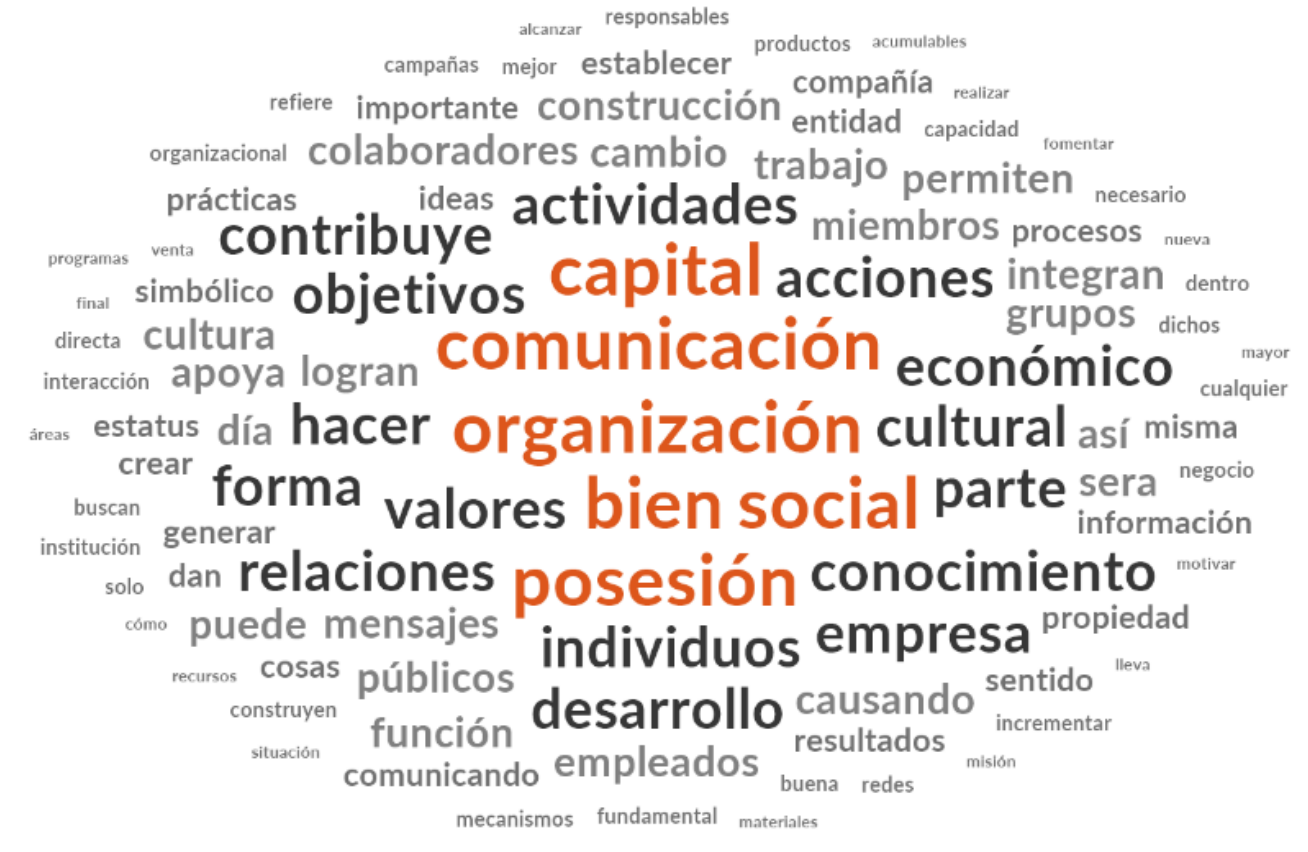

FIGURA 6. NUBE DE TEXTO SOBRE CAPITALES DE INFORMANTES CON ESTUDIOS DE LICENCIATURA

FUENTE: ELABORACIÓN PROPIA. 
Para los entrevistados con niveles de estudio de posgrado, como se observa en la Figura 7, los capitales están principalmente definidos como información o bien, y de manera secundaria con el conocimiento, actividades, objetivos, acciones, relaciones y personas. Destaca que las palabras económico y social sí aparecen de manera secundaria, pero no así la referencia a la cultura o lo cultural. En este sentido, parece que el nivel de estudios de posgrado genera que se conceptualicen los capitales más como un producto del conocimiento y la información que generan las personas a través de sus relaciones, acciones y el apego al logro de sus objetivos y que ciertamente podría ser compartido por medio de la comunicación.

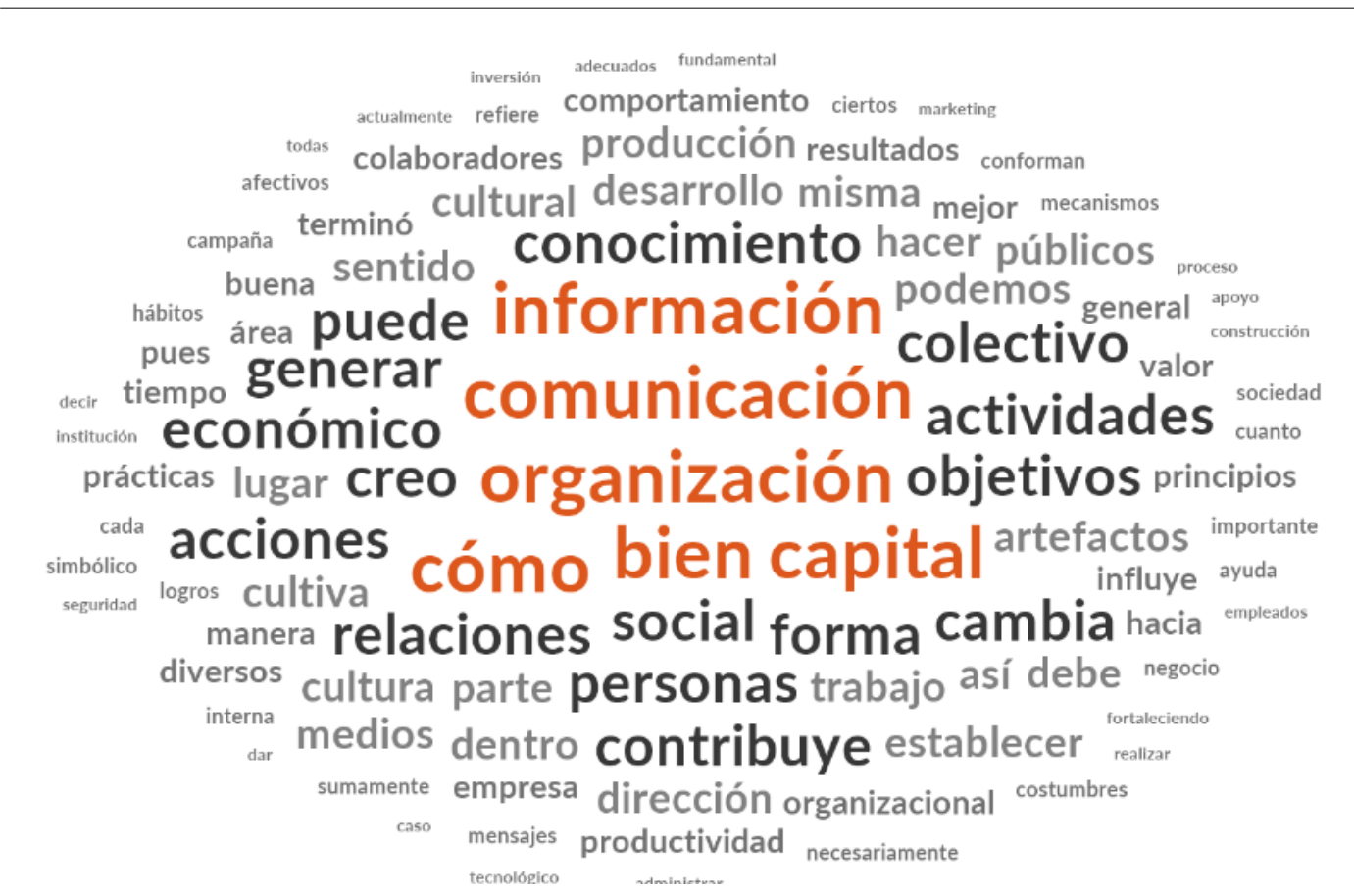

FIGURA 7. NUBE DE TEXTO SOBRE CAPITALES DE INFORMANTES CON ESTUDIOS DE POSGRADO

FUENTE: ELABORACIÓN PROPIA. 
En el análisis de las entrevistas considerando la especialidad de los entrevistados, aquéllos que la tienen en comunicación (Figura 8) definen a los capitales con base en bienes, objetivos, posesión y el verbo hacer de manera primordial, seguido de referentes como relaciones, actividades, acciones, conocimiento, individuo y verbos como desarrollar, poder, contribuir, apoyar y producir. En este sentido, la formación en comunicación pone en el centro a los objetivos y la necesidad de posesión y de bienes que pueden ser producto de relaciones y todas aquellas actividades o acciones de apoyo o en las que pueden contribuir desde la comunicación organizacional, aunque destaca el referente de poder que se relacionaría con la posesión.

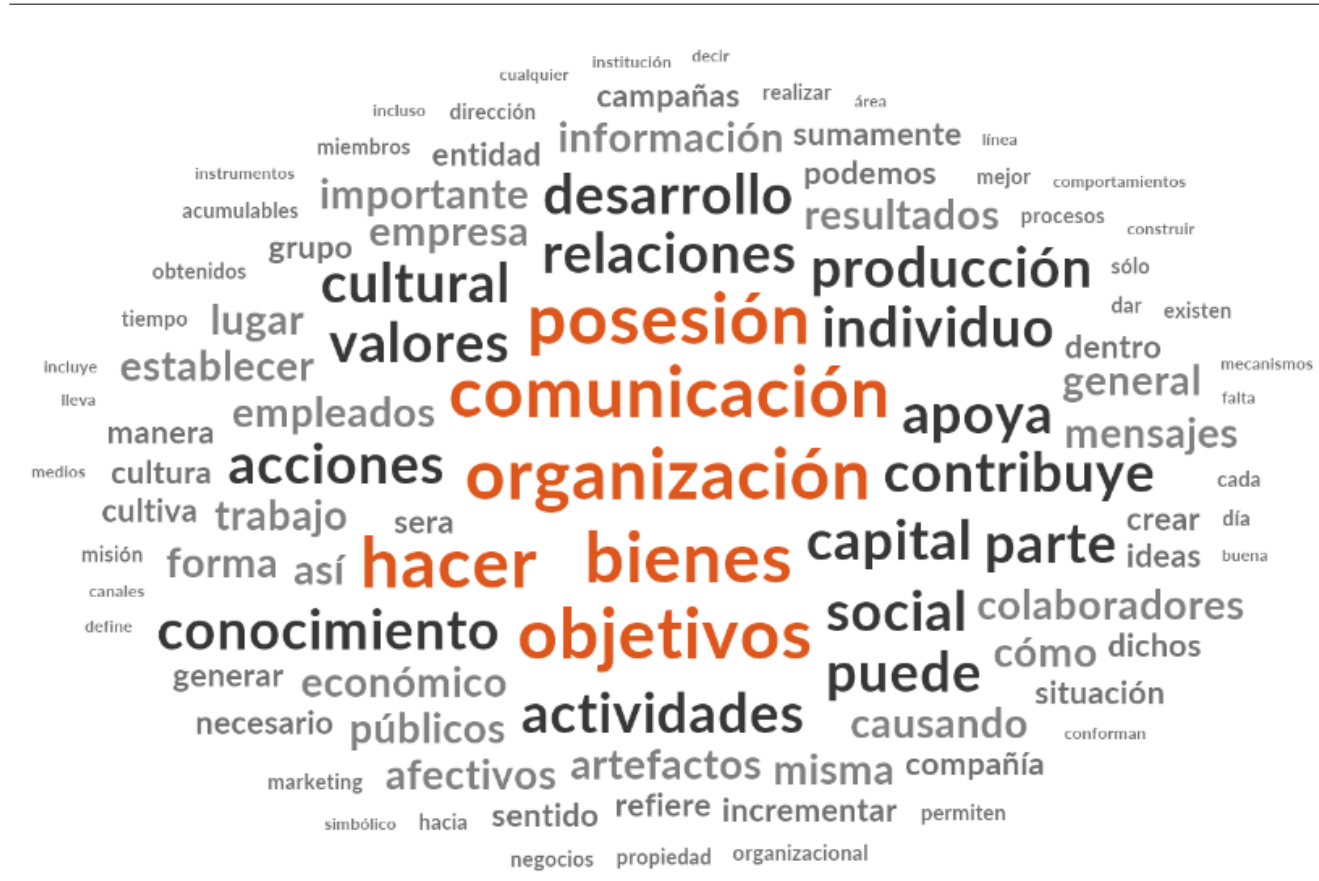

FIGURA 8. NUBE DE TEXTO SOBRE CAPITALES DE INFORMANTES CON ESPECIALIDAD EN COMUNICACIÓN

FUENTE: ELABORACIÓN PROPIA. 
Por su parte, los entrevistados que tienen especialidad en otras áreas o disciplinas (Figura 9) definieron los capitales en términos de un bien y las palabras social y persona, teniendo como referentes secundarios la información, el conocimiento, los activos, las relaciones, las cosas, la cultura, el valor, la forma y los verbos hacer, cambiar y formar. En el caso de este grupo de entrevistados llama la atención que el centro esté en las personas y en el bien, en lo social, que se visualiza como la forma, el hacer, las relaciones y el cambio; para ellos parece que no es importante que su trabajo en comunicación organizacional sea el resultado de apoyo o de su contribución, sino de sus conocimientos e información.

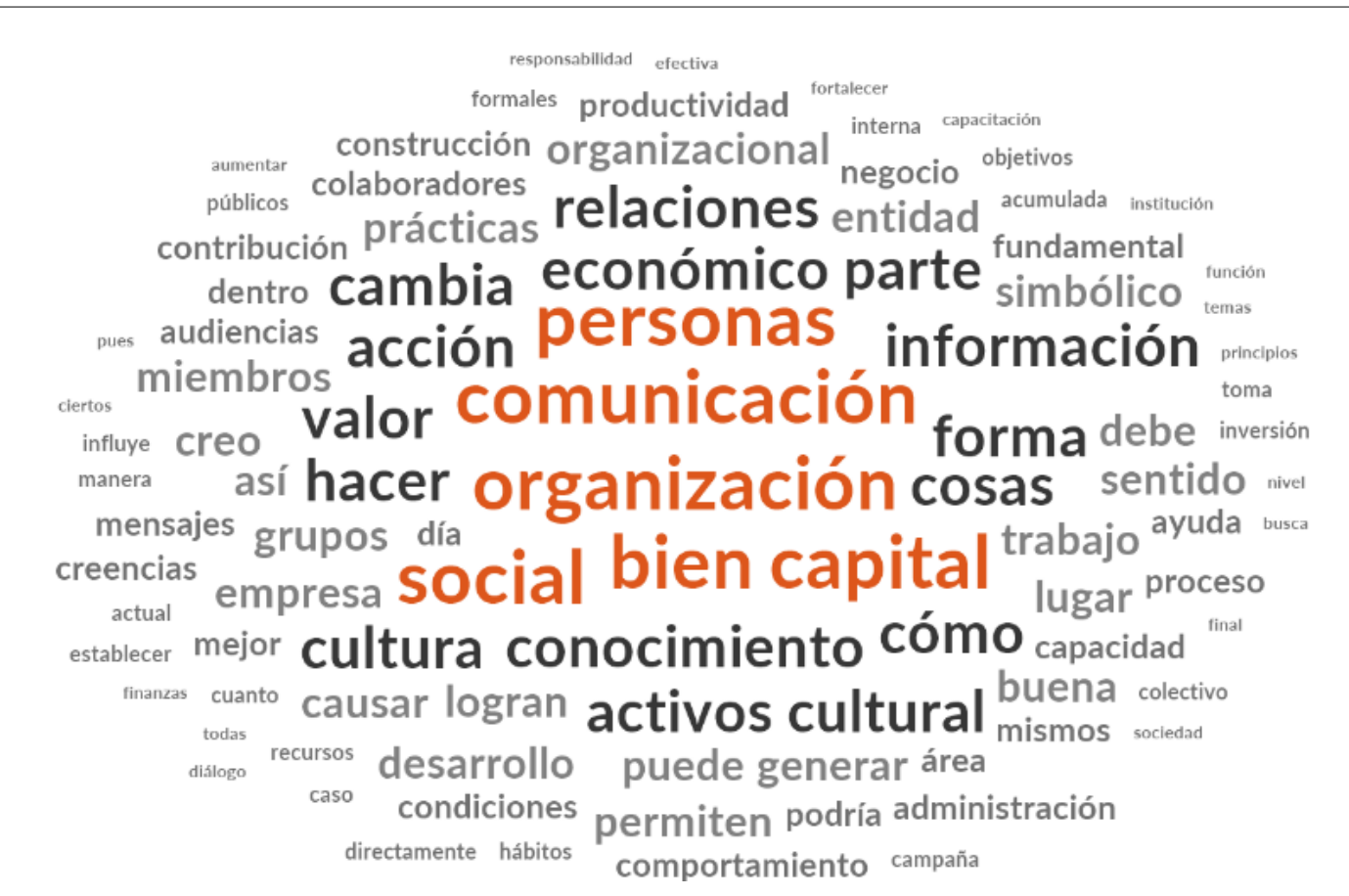

FIGURA 9. NUBE DETEXTOSOBRE CAPITALES DE INFORMANTES CON ESPECIALIDADEN OTRAS ÁREAS O DISCIPLINAS

FUENTE: ELABORACIÓN PROPIA. 
En cuanto a los informantes que ejercen como consultores en comunicación organizacional (Figura Io) se encontró que para ellos la definición de capitales se centra en las personas, la posesión y como un bien, refiriéndose de manera secundaria a la cultura o cultural, lo social, lo económico, las relaciones, el conocimiento, los objetivos, las actividades, los grupos, las acciones y el valor, principalmente. En este grupo de profesionales dedicados a la consultoría podría parecer lo indicado que primero que nada se centraran en el conocimiento que como expertos brindan en su función de asesores o guías, sin embargo, destacan la mención a las personas, es posible que como una referencia a la necesidad de que sus ideas se retomen como suyas por parte de las personas que conforman la organización a la que sirven, pues sin ello su trabajo se nulificaría.

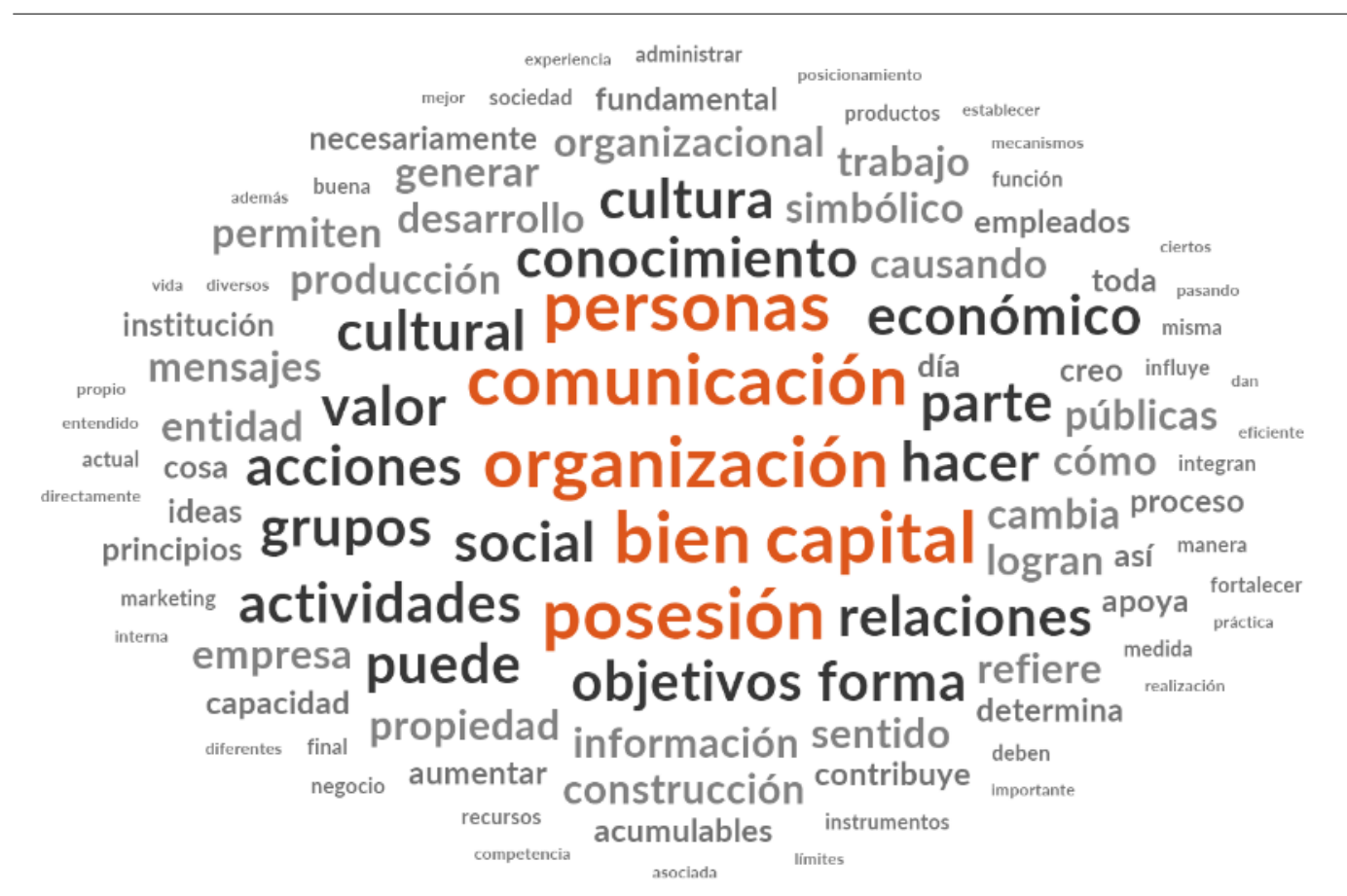

FIGURA 10. NUBE DE TEXTO SOBRE CAPITALES DE CONSULTORES EN COMUNICACIÓN ORGANIZACIONAL

FUENTE: ELABORACIÓN PROPIA. 
$\mathrm{Al}$ respecto de los capitales, los entrevistados que son académicos en el área de Comunicación organizacional (Figura II) se refirieron en primera instancia al verbo establecer y la palabra relaciones, independientemente de mencionar explícitamente capital, social y económico; y en segunda instancia a empleados, artefactos, humanos, información, objetivo, públicos, manera y acción, además de los verbos cuenta, será, realizar y conocemos. En este sentido, la formación académica que tienen en sus manos según lo que se refleja en los entrevistados se centra en la importancia de establecer relaciones, alineadas a objetivos, pensando en los públicos y con base en acciones y artefactos, por sintetizar algunas de las principales ideas centrales. Si esta consideración es correcta, la comunicación desde su punto de vista podría interpretarse como aquella que aporta la habilidad a la organización de relacionarse con los públicos para lograr sus objetivos.

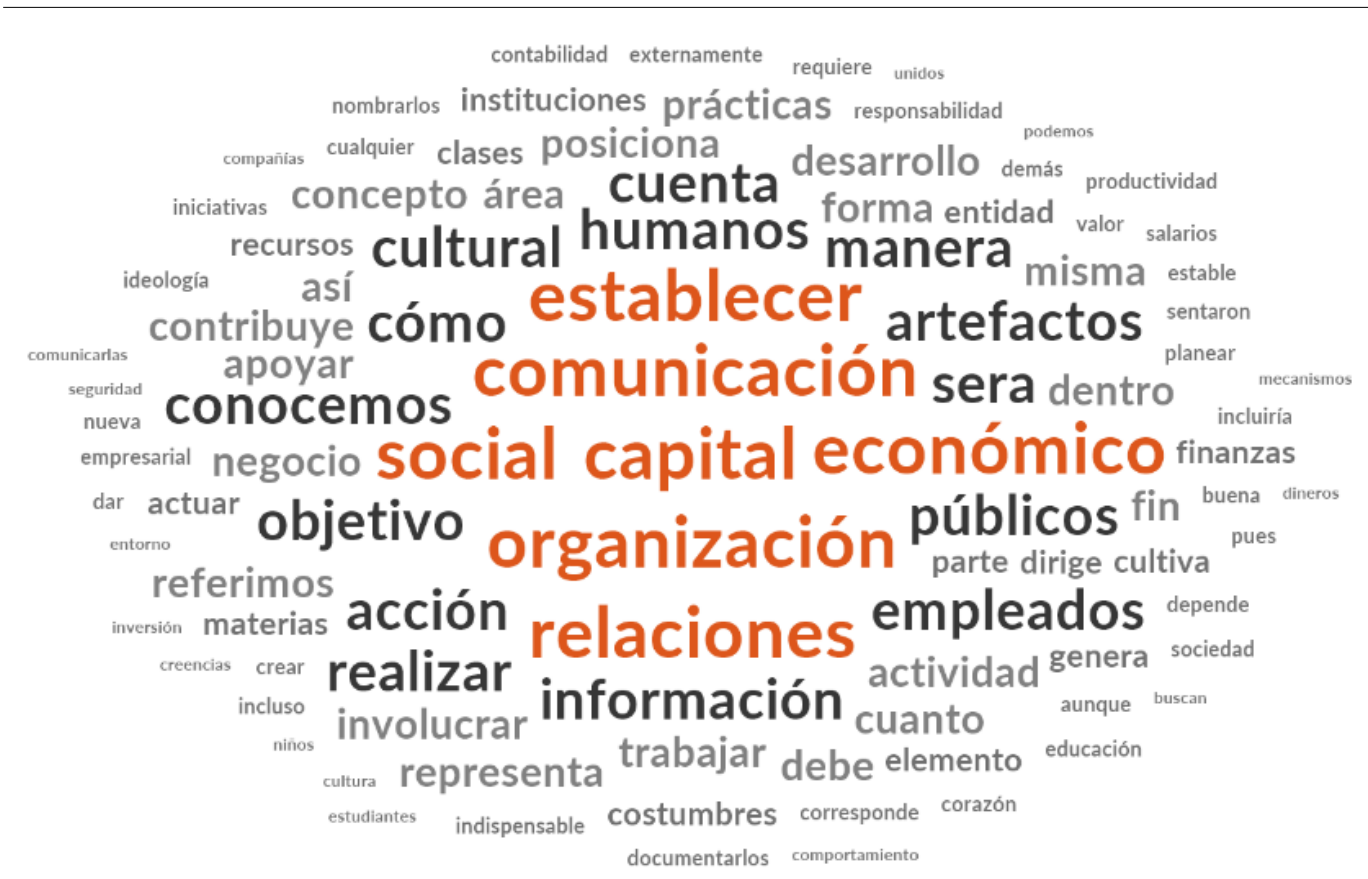

FIGURA 11. NUBE DE TEXTO SOBRE CAPITALES DE ACADÉMICOS EN COMUNICACIÓN ORGANIZACIONAL

FUENTE: ELABORACIÓN PROPIA. 
Los informantes que se desempeñan como profesionales de la comunicación dentro de alguna organización definieron los capitales (Figura I2) con palabras como individuos, objetivos, y la referencia a las palabras bien y capital explícitamente, además de mencionar las relaciones, lo social, los colaboradores, públicos, miembros a la par de las acciones, actividades, conocimiento con verbos como formar, crear, trabajar, contribuir y generar. Desde esta perspectiva, la comunicación puede entenderse como un bien que trabaja desde los individuos para generar bienes y relaciones que entrega a otros individuos (públicos, colaboradores, miembros). Con base en esta interpretación, la comunicación es un enlace que posibilita que las personas trabajen para las personas y en ello generen capital social y económico (no se hizo referencia al capital cultural hasta un nivel más debajo de menciones) en relación con los objetivos planteados.

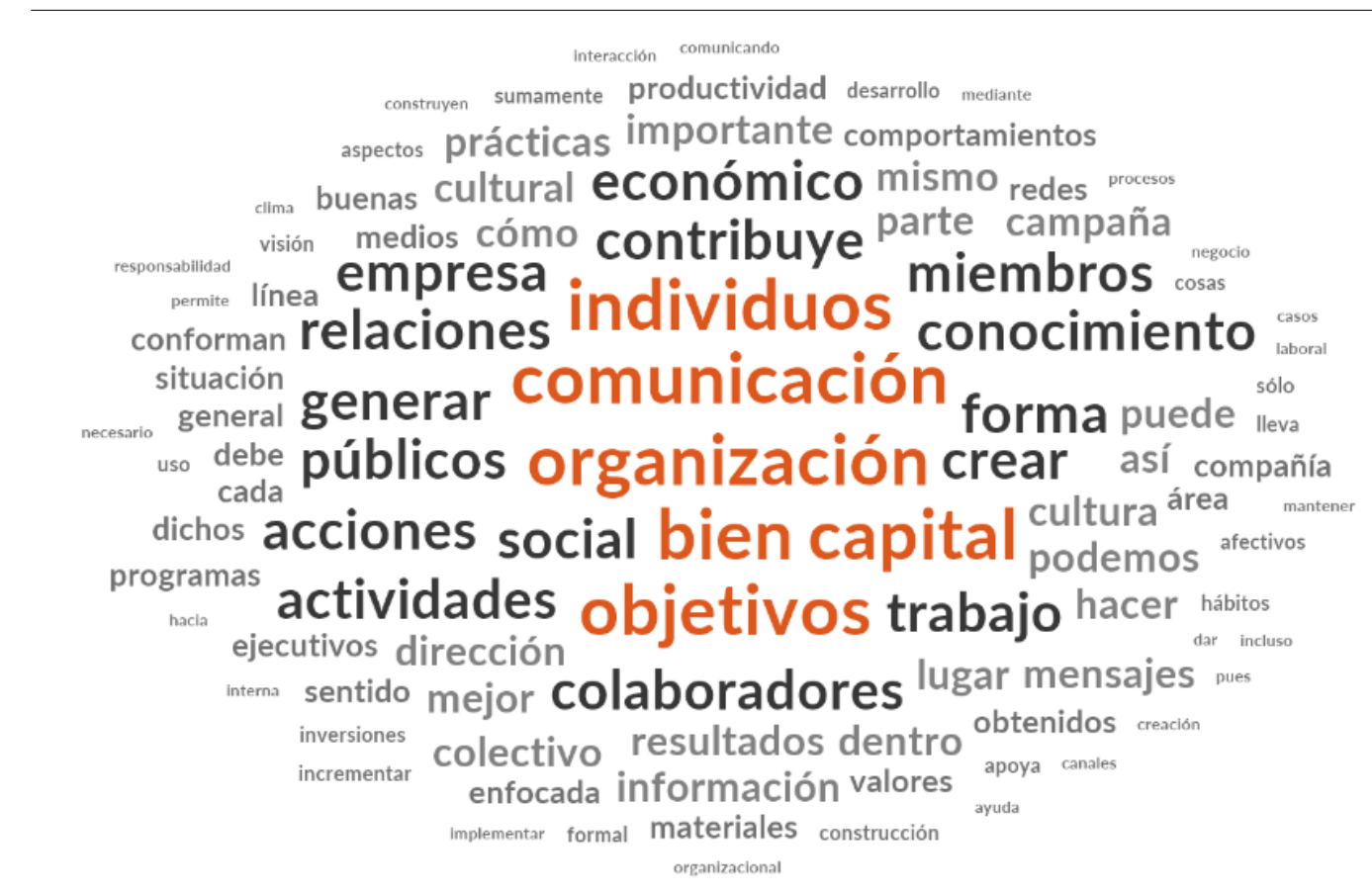

FIGURA 12. NUBE DE TEXTO SOBRE CAPITALES DE PROFESIONALES EN COMUNICACIÓN ORGANIZACIONAL

FUENTE: ELABORACIÓN PROPIA. 


\section{ANÁLISIS Y DISCUSIÓN}

Como puede verse a lo largo de los resultados del análisis textual, contextual e interpretativo, la conceptualización de los distintos tipos de capital es diferenciada no solo por el género, el nivel o grado máximo de estudios y la especialidad, sino que incluso dentro de la concepción más profunda de cómo se concibe cada capital en términos de contar con lo necesario para ejercer un determinado tipo de poder o dominio (Bourdieu, 1997) dentro del campo y con ello tener elementos que a la luz de lo que se espera de un profesional, académico o consultor de la comunicación signifique un elemento de valor a los ojos de los otros (Bourdieu, 1997a). Las diferentes ubicaciones que tiene el referente de persona, individuo y relaciones, ponen de manifiesto que se trata de un componente que sostiene la construcción de los capitales y a las personas mismas en sus redes (Bourdieu, 2007b).

En el caso del capital cultural llama la atención que se presente en los entrevistados sin importar el género ni la especialidad, pero en mayor medida dentro de quienes tienen estudios de licenciatura y no así dentro de los de posgrado. No se encontró ninguna mención de tipo prioritaria a este tipo de capital entre los profesionales en comunicación organizacional, aunque sí aparece en segundo nivel entre los consultores y académicos. En este ámbito de la cultura o lo cultural es posible que se requiera un mayor énfasis en su valor intrínseco como parte del mercado de bienes simbólicos y la forma en que este se reproduce en las distintas clases sociales (Bourdieu y Passeron, 1995). En cuanto al capital económico, entendido de origen como los recursos monetarios y financieros (Bourdieu, 2003), destaca su mención prioritaria entre los entrevistados de género masculino, académicos y con especialidades distintas a la comunicación, sin importar su grado de estudios. Mientras que aparece en segundo lugar para consultores y profesionales de comunicación organizacional. En este sentido es posible que los especializados en comunicación no tienen entre sus prioridades el capital económico ni en el sentido de la capacidad de movilizarlo (Bourdieu, 2007b) e incluso convertirlo en capital simbólico que se pueda reproducir y permanecer bajo la conceptualización de relaciones (Bourdieu, 2007). Finalmente, en cuanto al capital social, destaca que se haya ubicado entre las principales menciones de los entrevistados de género masculino, de licenciatura, académicos y con especialidades distintas a la comunicación. Llama la atención que el capital social como fuente principal del valor que se puede movilizar por las redes o relaciones no fuera mencionado de manera prioritaria por los entrevistados con especialidad en comunicación, los que cuentan con estudios de posgrado, ni por las entrevistadas de género femenino, aunque en los tres casos aparece la referencia al término relaciones. Es decir, posiblemente se valoren más las relaciones o redes que se requieren para la formación de capital, en cualquiera de sus tres tipos analizados, y no únicamente en el caso del capital social. 


\section{CONCLUSIONES}

En esta investigación se pudo constatar que existe una visión compartida sobre el capital cultural entre los profesionales, consultores y académicos de la comunicación que coincide sin importar su especialidad de estudios ni su género; al igual que sobre el capital económico sin importar su grado de estudios. Sin embargo, destaca el hecho de que no hubo coincidencias en la forma de definir el capital social entre los entrevistados. Los entrevistados de género masculino dieron mayor peso, en su verbalización, al capital económico y al capital social, mientras que los entrevistados con grado de estudios de licenciatura hicieron mayores referencias al capital cultural y al capital social. Finalmente, los entrevistados que tienen especialidades de estudio distintas a la comunicación hicieron mención principalmente del capital económico y social. Estos resultados nos permiten inferir que la mayor preocupación entre quienes ejercen funciones de comunicación desde la consultoría está en contar con el apoyo de las personas, entre los académicos se encuentra establecer relaciones y entre los profesionales en comunicación organizacional dentro de las organizaciones se encuentran los individuos y sus objetivos.

Los retos que enfrenta la comunicación organizacional ante los efectos de la crisis sanitaria, económica, política y social de los años recientes, requerirá un trabajo arduo para comprender de manera unívoca al capital social principalmente, pero también el cultural y económico, de manera que sea posible contribuir desde las personas para las personas en la construcción de valor con base en su conocimiento y en su experiencia y así generar relaciones de amplio alcance enfocadas al bien común que tanto requiere la sociedad a nivel local, regional y global.

\section{REFERENCIAS}

Ackoff, R. (2000). Recreación de las corporaciones. Un diseño organizacional para el siglo XXI. Oxford University Press.

Ackoff, R. (2005). Rediseñando el futuro. Limusa.

Barquero, J.y Castillo-Esparcia, A. (20II). Marco teórico y práctico de las relaciones públicas. Furtwangen Editores: McGraw Hill.

Bourdieu, P. (2003). La distinción: Criterio y bases sociales del gusto. Taurus.

Bourdieu, P. (2007). La miseria del mundo. Fondo de Cultura Económica.

Bourdieu, P. (2002). Campo de poder, campo intelectual: Itinerario de un concepto. Montressor.

Bourdieu, P. (2003a). Cuestiones de sociología. Istmo. 
Bourdieu, P. (2007b). El sentido práctico. Siglo XXI.

Bourdieu, P. (I997a). Espacio social y espacio simbólico. En P. Bourdieu: Razones prácticas: Sobre la teoría de la acción. Anagrama, pp. II-26.

Bourdieu, P. (1997). Meditations pascaliennes. Ed. du Seuil.

Bourdieu, P. (1996). Razones prácticas sobre la teoría de la acción. Editorial Anagrama.

Bourdieu, P. (1996b). Fieldwork of philosophy. En P. Bourdieu, Cosas Dichas (pp. 17-43). Gedisa.

Bourdieu, P.y Passeron, J-C. (1995). La reproducción: elementos para una teoría del sistema de enseñanza. Fontamara.

De Salas, M. y Pérez, R. (20ı). La aplicación de la nueva teoría estratégica al campo de la comunicación empresarial: el hombre relacional clave en el actual posicionamiento de marca. Fisec Estrategias, V(13), pp. 49-62.

Etkin, J. (2006). Gestión de la complejidad en las organizaciones. La estrategia frente a lo imprevisto y lo impensado. Ediciones Granica.

Grunig, J. y Grunig, L. (20II). Characteristics of Excellent Communication. En T. Gillis: IABC Handbook of Organizational Communication: A Guide to Internal Communication, Public Relations, Marketing, and Leadership. Hoboken: Jossey-Bass, pp. 3-I4.

Grunig, J., Grunig, L. y Dozier, D. (2002). Excellent Public Relations and Effective Organizations: A Study of Communication Management in Three Countries. Lawrence Erlbaum Associates, Inc.

Katz, D. y Kahn, R. L. (I966). The social psychology of organizations. Wiley.

Llano, C. (1997). Dilemas éticos de la empresa contemporánea. Fondo de Cultura Económica.

Esta obra está bajo Licencia Creative Commons Atribución-NoComercial-SinDerivadas 4.o Internacional. 